\title{
Space Charge Behavior under Thermal Gradient in Cross-Linked Polyethylene and Ethylene-Propylene Rubber
}

\author{
Hiroaki Uehara, ${ }^{*}$ Zongze Li, ${ }^{1}$ Qin Chen, ${ }^{2}$ Gian Carlo Montanari, ${ }^{3}$ and Yang Cao ${ }^{1}$ \\ Department of Electrical and Electronic Engineering, Kanto Gakuin University, \\ 1-50-1 Mutsuura-higashi, Kanazawa-ku, Yokohama, Kanagawa, 236-8501, Japan \\ ${ }^{1}$ Department of Electrical and Computer Engineering, University of Connecticut, \\ 97 North Eagleville Road, Storrs, CT 06269-3136, USA \\ ${ }^{2}$ GE Global Research Center, 1 Research Circle, Niskayuna, NY 12309, USA \\ ${ }^{3}$ Department of Electrical, Electronic and Information Engineering, University of Bologna, \\ Viale Risorgimento 2, 40136 Bologna, Italy
}

(Received December 5, 2016; accepted May 11, 2017)

Keywords: space charge, high-voltage direct current (HVDC), thermal gradient, cross-linked polyethylene (XLPE), ethylene-propylene rubber (EPR), DC stress

The direct observation of space charge injection, transport, accumulation, and recombination in solid dielectrics, as well as their spatial evolution over time, is of considerable importance in the investigation of design stress and the aging mechanism of engineering dielectrics under a high electric field. In a high-voltage direct current (HVDC) power cable with an expected temperature gradient at operating temperature and electric field, the spatial variation of the ratio of two electromagnetic constitutive parameters of the cable dielectric, the permittivity vs conductivity, acquires the presence of space charge across the insulation. Such a space charge distribution depends strongly on the temperature and electric field coefficients of these electromagnetic constitutive parameters. In addition, the injection of charges across the conductor/ insulation interface and the subsequent transport, trapping and detrapping of these charges over various chemical and physical defects further enhance the electric field distortion and aging of cable dielectrics. Numerous studies have been conducted under a parallel-plate configuration to understand such a space charge effect. However, nearly all of them were conducted under uniform isothermal conditions. The ability to perform a space charge profiling study under a thermal gradient in the development phase of new extruded high-voltage direct current cable materials brings in the most value upstream. In this study, through model-aided design, a parallel-plate, pulsed-electroacoustic (PEA) space charge profiling technique is extended to include a thermal gradient in flat dielectric samples. The space charge behavior and dynamics within flat dielectric specimens in the presence of a thermal gradient have been studied extensively through a modified PEA system to provide insights into the high-field aging mechanisms of new materials developed for energy-efficient power devices and renewable integration.

\footnotetext{
"Corresponding author: e-mail: huehara@kanto-gakuin.ac.jp
} http://dx.doi.org/10.18494/SAM.2017.1538 


\section{Introduction}

High-voltage direct current (HVDC) and flexible AC have been adopted widely for longhaul bulk grid interconnections, urban grid decongestions, island electrification, and renewable integrations. In particular, HVDC provides efficient nonsynchronized bulk electric power transmissions with the economic benefits of reduced power loss, no reactive power, easy connection, and enhanced stability. ${ }^{(1,2)}$ Free from reactive power loss, HVDC cables become viable solutions for submarine power transmission, off-shore wind power integration, and harsh environment electrification. While mass-impregnated (MI) or oil-filled (OF) cables have achieved long proven service reliability, extruded HVDC cable insulation free from the environmental risk of oil leakage is preferred owing to the ease of manufacturing, maintenance, field deployment, and simplified accessory design and procurement.

Most of the extruded cable insulations today are based on cross-linked polyethylene (XLPE) and ethylene-propylene rubber (EPR). ${ }^{(3)}$ Extruded HVDC cables today are based on modified XLPE. The major challenge for HVDC cabling resides in the insulation. Space charge accumulation in DC cable insulation will result in local electric field enhancement and reduction in DC electric strength. This is especially significant during voltage polarity reversal (required for changing the direction of power flow) in line-commutated converter (LCC) systems. With the introduction of a voltage source converter (VSC)-based HVDC converter station where polarity reversal is not required, the use of the extruded DC cable based on XLPE has been increased rapidly due to the reduction in performance deterioration by space charge accumulation. With the growing demand for HVDC rating in both power and voltage as well as a new system topology in such multiterminal HVDC, further optimization of DC insulation is still greatly needed for enhanced performance and reliability.

The charge injection, transport, accumulation, and recombination of space charge in extruded cable insulation, such as XLPE or EPR, deteriorate the insulating performance under HVDC. ${ }^{(4,5)}$ Therefore, it is essential to clarify the formation mechanism and its dependence on the aging of space charge. ${ }^{(6-8)}$ In general, the temperature dependence of the electrical properties of insulating materials in HVDC power cables affects all aspects of the formation of space charges, which often develop complicated charge patterns that evolve over time. When the load current flows through the transmission cable, the thermal gradient is generated by Joule heating in the cable insulator. Furthermore, the electrical conductivity of typical polymeric insulating materials depends strongly on the temperature and hence the radius position across the transmission cable. ${ }^{(9)}$ Under cylindrical coordinates, the radial spatial variation of the ratio of two electromagnetic constitutive parameters of the cable dielectric, the permittivity vs conductivity, acquires the presence of the space charge $\rho$ across the insulation. ${ }^{(10)}$

$$
\rho=j \cdot \nabla\left(\frac{\varepsilon}{\sigma}\right)
$$

Here, $j$ is the current density and $\varepsilon$ and $\sigma$ are the two electromagnetic constitutive parameters of the cable dielectric, namely, permittivity and conductivity, respectively. Such a space charge distribution depends strongly on the temperature and electric field coefficients of these electromagnetic constitutive parameters. In addition, the injection of charges across the conductor/ insulation interface and the subsequent transport, trapping, and detrapping of these charges over 
various chemical and physical defects further enhance the electric field distortion and aging of cable dielectrics.

The studies of temperature dependence on space charge have been conducted by the following researchers. Fukuma et al. reported space charge measurements at a uniform temperature of up to $90{ }^{\circ} \mathrm{C}^{(11)}$ Choo et al. investigated thermal gradient effects using actual cables. ${ }^{(12)}$ Chen et al. controlled the temperatures of upper and lower electrodes using oil and water, and used their improved equipment in which a temperature gradient can be formed. ${ }^{(13)}$ Kadowaki et al. indicated that there was a remarkable polarity effect in the long-time range breakdown under a temperature gradient. ${ }^{(14)}$ Most of these studies were conducted under isothermal conditions or require the construction of a prototyping cable for thermal gradient generation under a cylindrical configuration. However, the ability to perform a space charge profiling study under a thermal gradient in the development phase of new extruded HVDC cable materials brings in the most value upstream. This necessitates the space charge study for flat coupons as in the early development phases, and only lab-scale quantities of experimental new formulations are available for DC performance characterizations.

In this study, through model-aided design, a parallel-plate, pulsed-electroacoustic (PEA) space charge profiling technique is extended to thermal gradient generation across flat dielectric samples. The space charge behavior and dynamics within flat dielectric specimens in the presence of a thermal gradient have been studied extensively by using this modified PEA for two model XLPE and EPR specimens.

\section{Materials and Methods}

\subsection{Sample preparation}

In this study, model XLPE and EPR based on DC insulation were hot-pressed into plaques of $4 \times 4 \mathrm{~cm}^{2}$ size and 200-330 $\mu \mathrm{m}$ thickness. All specimens were metallized with $80 / 20 \mathrm{wt} \% \mathrm{gold} /$ palladium electrodes of $1 \mathrm{~cm}$ diameter on both sides. Due to the impedance mismatch on the side of high-voltage electrode, the charge injection and charge amount may be suppressed slightly by metallized gold/palladium. For sample loading in a PEA cell, a torque of $15 \mathrm{~N} \cdot \mathrm{m}$ was applied to all samples to ensure good contacts and uniform application of pressure.

\subsection{Experimental procedure}

Figure 1 shows schematically the modified PEA system based on a Techimp PEA flat coupon module by incorporating electric heaters for thermal gradient generation. The design represents a minimal and simple modification of the existing PEA system while a uniform thermal gradient across a flat sample could be achieved. In detail, thin-film electric heaters were designed and incorporated to heat up the bottom aluminum electrode with sufficient power to generate an adequate thermal gradient across the sample. A feedback loop was provided for proportionalintegral-derivative (PID) temperature control by deploying resistance-temperature detectors (RTDs) for temperature monitoring for both the upper and lower surfaces of the specimen. The ambient temperature was maintained at room temperature (RT). If needed, the whole PEA cell can be enclosed in an environmental chamber for different ambient temperatures. 


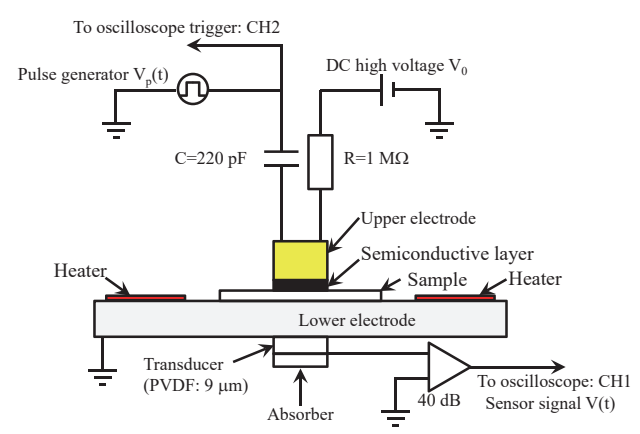

Fig. 1. (Color online) Modified PEA system with electric heaters.

The acoustic signal generation was accomplished through the application of high-voltage impulses with a rise time of $\sim 1 \mathrm{~ns}$, a peak voltage of $350 \mathrm{~V}$, and a repetition rate of $150 \mathrm{~Hz}$ to flat samples under HV biasing and thermal gradient. The acoustic waves were detected by a polyvinylidene fluoride (PVDF) film sensor of $9 \mu \mathrm{m}$ thickness.

In an actual polymeric HVDC transmission cable, the electric field is designed to be $10-30 \mathrm{kV} /$ $\mathrm{mm}$. Therefore, film samples were mostly studied under a DC field of $20 \mathrm{kV} / \mathrm{mm}$. Moreover, in this study, a higher electric field of $100 \mathrm{kV} / \mathrm{mm}$ for XLPE and EPR was also employed to facilitate the mechanism study of space charge injection, transport, accumulation, and recombination phenomena under extreme conditions. The measurement time of the space charge was $10800 \mathrm{~s}(3 \mathrm{~h})$. In the case of $100 \mathrm{kV} / \mathrm{mm}$ application, the measurement time was $1000 \mathrm{~s}$.

\section{Results}

\subsection{Finite element simulation of thermal gradient in sample using COMSOL}

The thermal gradient in actual power cables is typically $\sim 1 \mathrm{~K} / \mathrm{mm}$. For a flat sample of $<1$ $\mathrm{mm}$ thickness, precise and uniform temperature gradient control has to be carefully realized. Figure 2 shows the finite element (FEM) simulation of a thermal gradient in a sample for this specific design at RT using COMSOL (2D axial symmetrical). Although the thermal gradient was $\Delta T=2.2 \mathrm{~K}$ in this simulation, the actual observed results are based on the study performed with a thermal gradient of $\Delta T=1.2 \mathrm{~K}$ across thin-film samples with an ambient temperature of RT.

Figure 3 depicts the concept of temperature drop across a series of thermal resistances, according to Fourier's law represented by Eqs. (2) and (3). Here, $q$ is the thermal flux.

$$
\begin{gathered}
q=\frac{T_{1}-T_{5}}{R_{A}+R_{B}+3 R_{C}} \\
\Delta T=q R_{A}
\end{gathered}
$$




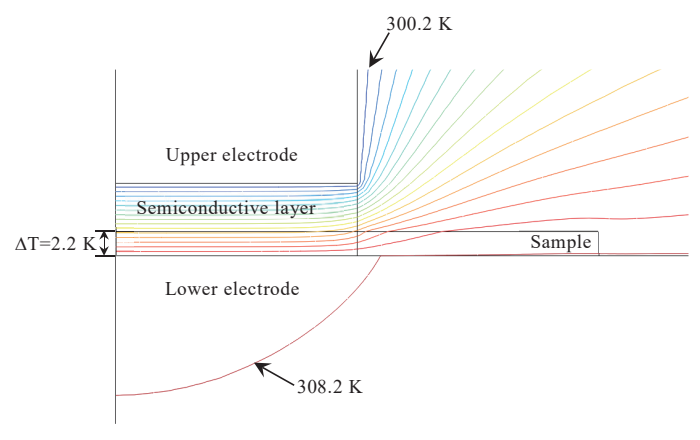

Fig. 2. (Color online) FEM simulation of thermal gradient in sample for this specific design at RT using COMSOL (2D axial symmetrical).

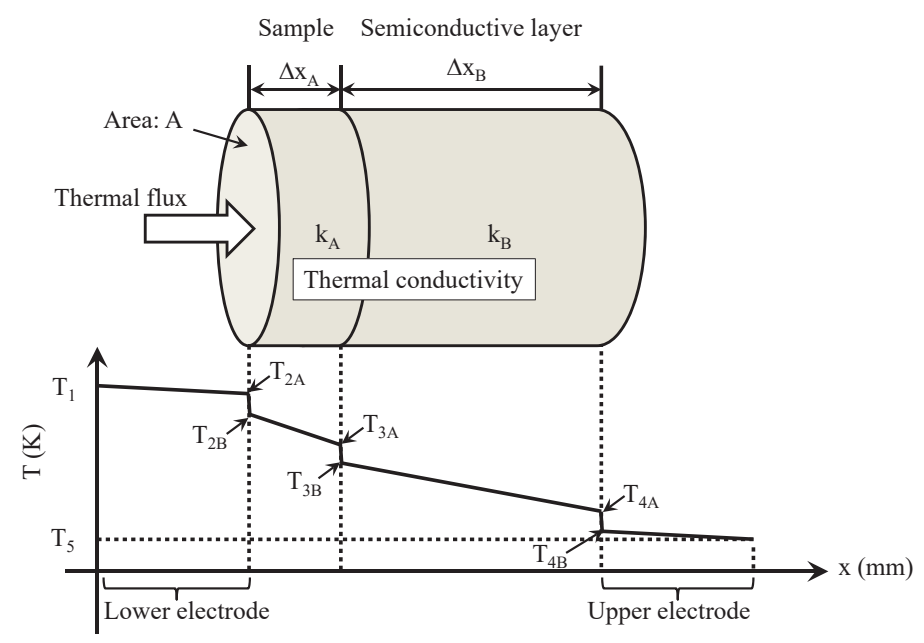

Fig. 3. (Color online) Concept of temperature drop governed by Fourier's law.

The temperatures of the two electrodes are $T_{1}$ and $T_{5}$, respectively. The area of the contact surface is $A$. The thicknesses of the sample and semiconductive layer are $\Delta x_{A}=200 \mu \mathrm{m}$ and $\Delta x_{B}=1 \mathrm{~mm}$, respectively. The thermal conductivities of the sample and semiconductive layer are $k_{A}$ and $k_{B}$, the thermal resistances are $R_{A}=\Delta x_{A} / k_{A} A$ and $R_{B}=\Delta x_{B} / k_{B} A$, respectively, and the contact thermal resistance is $R_{c}=1 / h_{c} A, h_{c}$ being the contact thermal conductance. With the above parameters, the simulated temperature drop across the sample is $\Delta T=1.3 \mathrm{~K} .{ }^{(13,15)}$ Here, the temperature drop at the $\mathrm{Al}$ electrode is ignored because of its high thermal conductivity. The thermal conductivities of XLPE, EPR, and the semiconductive layer are the same at $0.3 \mathrm{~W} / \mathrm{m} \cdot \mathrm{K}$. Moreover, the contact thermal resistance is estimated as $0.2 \mathrm{~m} \cdot \mathrm{K} / \mathrm{W}$. The resulting thermal gradient of $\Delta T=1.3 \mathrm{~K}$ is almost the same as the actual thermal gradient of $\Delta T=1.2 \mathrm{~K}$ measured using RTD sensors. In addition, as revealed by FEM simulation, the planar temperature distribution within the sample is highly uniform. 


\subsection{Comparison of space charge behavior at $20 \mathrm{kV} / \mathrm{mm}$ in XLPE without and with thermal gradient}

Figures 4 and 5 show the space charge distributions and corresponding electric fields in XLPE at RT under a DC stress of $20 \mathrm{kV} / \mathrm{mm}$ without and with a thermal gradient, respectively. Without a thermal gradient, the charge accumulation was not observed within the first $1000 \mathrm{~s}$. After 1000 $\mathrm{s}$, heterocharge accumulation and field distortion were observed during the extended application of DC stress to 10800 s, as shown in Figs. 4(a) and 5(a).

On the other hand, with a thermal gradient, although the charge accumulation was not evident up to $1000 \mathrm{~s}$, more heterocharges were observed to accumulate. Positive charges accumulating in the vicinity of $100 \mu \mathrm{m}$ and negative charges accumulating in the vicinity of $200 \mu \mathrm{m}$ can be seen in Fig. 4(b). The field distortion due to the space charge accumulation can also be seen in Fig. 5(b). Moreover, a slight peak shift in charge distribution has occurred with a thermal gradient, as shown in Fig. 4(b). ${ }^{(16)}$

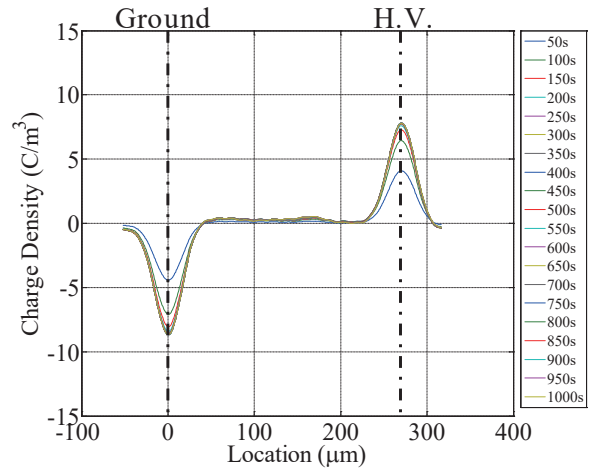

$0-1000 \mathrm{~s}$

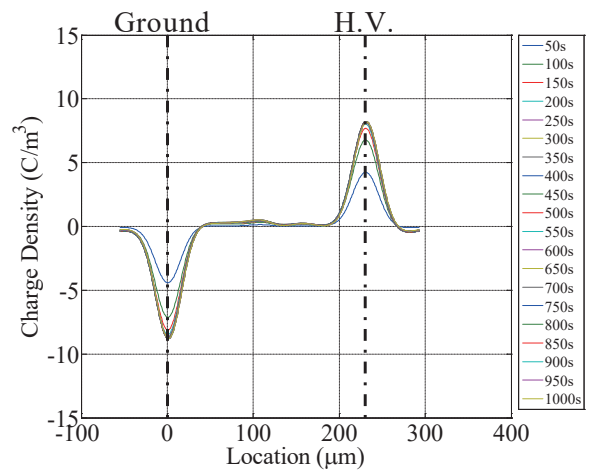

$0-1000 \mathrm{~s}$

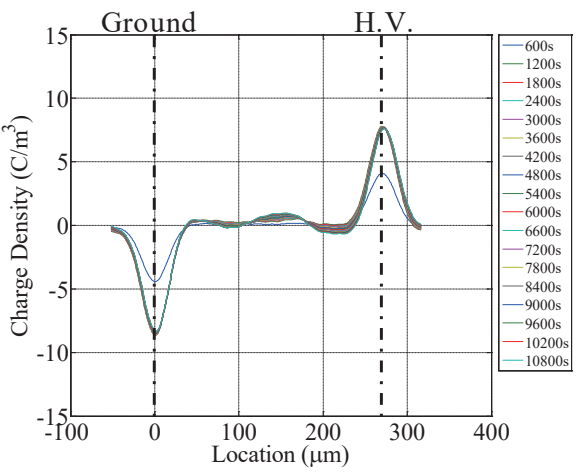

$0-10800 \mathrm{~s}$

(a)

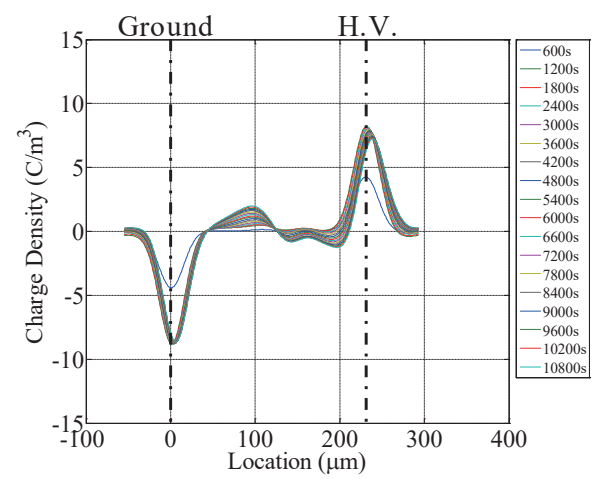

$0-10800 \mathrm{~s}$

(b)

Fig. 4. (Color online) Space charge distributions in XLPE at RT under DC stress of $20 \mathrm{kV} / \mathrm{mm}$ without and with thermal gradient (1000 and $10800 \mathrm{~s}$ ). (a) $\Delta T=0$ and (b) $\Delta T=1.2 \mathrm{~K}$. 


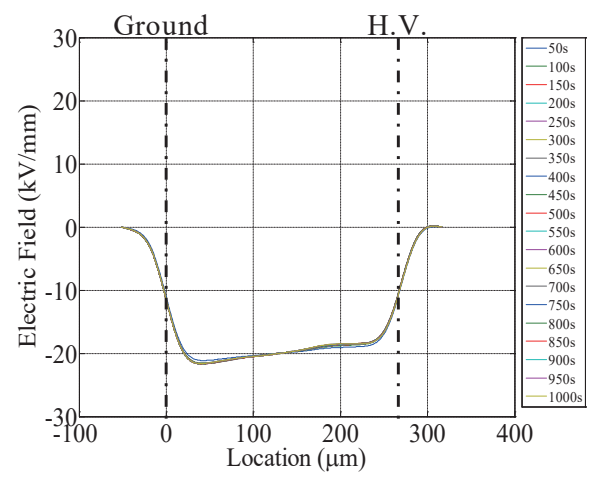

$0-1000 \mathrm{~s}$

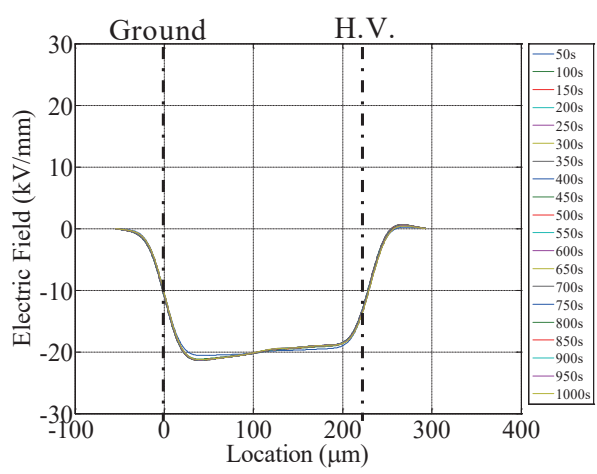

$0-1000 \mathrm{~s}$

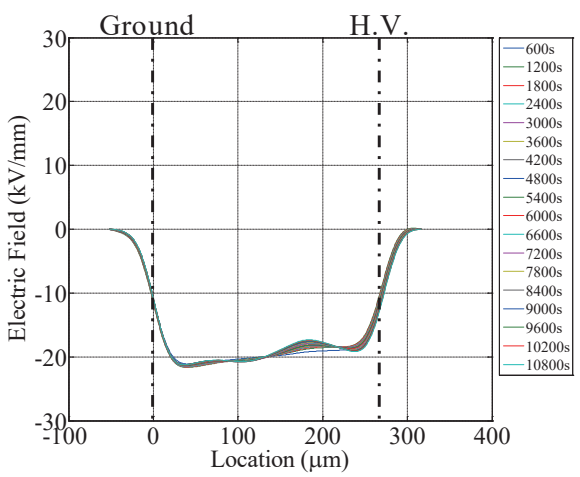

$0-10800 \mathrm{~s}$

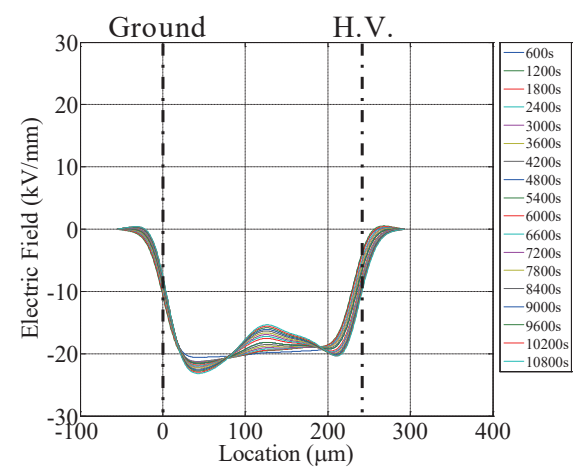

$0-10800 \mathrm{~s}$

(b)

Fig. 5. (Color online) Electric fields in XLPE at RT under DC stress of $20 \mathrm{kV} / \mathrm{mm}$ without and with thermal gradient (1000 and $10800 \mathrm{~s}$ ). (a) $\Delta T=0$ and (b) $\Delta T=1.2 \mathrm{~K}$.

\subsection{Comparison of space charge behavior at $20 \mathrm{kV} / \mathrm{mm}$ in EPR without and with thermal gradient}

Figures 6 and 7 show the space charge distributions and electric fields in EPR under a DC stress of $20 \mathrm{kV} / \mathrm{mm}$ without and with a thermal gradient, respectively. The charge density in the vicinity of both electrodes was slightly higher than that in XLPE, as shown in Fig. 6. Without a thermal gradient, heterocharges were observed even within $1000 \mathrm{~s}$, as shown in Fig. 6(a). In particular, the electric field in the vicinity of both electrodes was enhanced slightly, as shown in Fig. 7(a). Moreover, it can be seen that the heterocharge accumulation increased over time.

With a thermal gradient, heterocharges also accumulated even within the first $1000 \mathrm{~s}$, as shown in Fig. 6(b), and they further accumulated over time. Moreover, it appears that, over time, more homocharges were injected from the anode. A slight peak shift was also observed in the charge distribution in the presence of a thermal gradient, as in the case of XLPE. ${ }^{(16)}$ When homocharges were injected, the location of field distortion shifted to the inside of the bulk, as shown in Fig. 7(b). Note that the space charge accumulation and field distortion for both the XLPE and EPR under study are in similarly low magnitudes. 


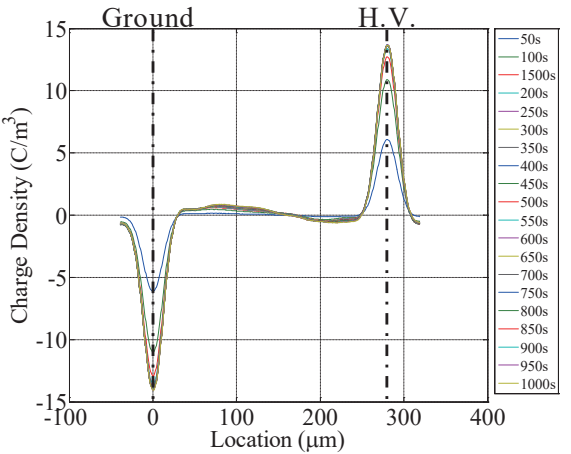

$0-1000 \mathrm{~s}$

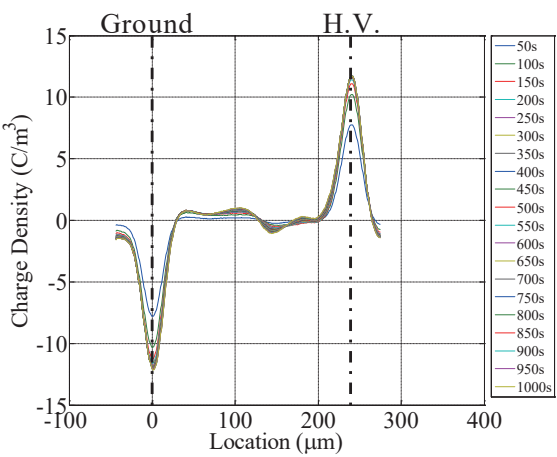

$0-1000 s$

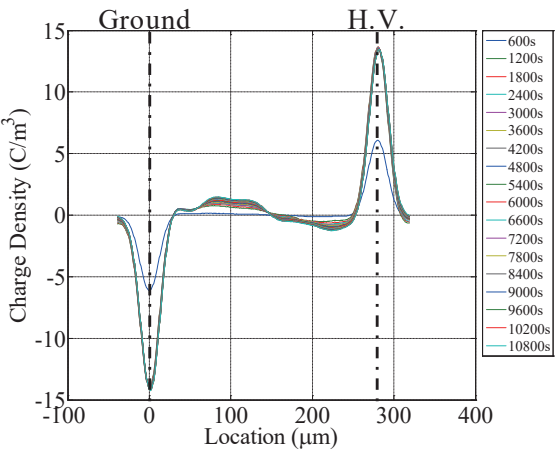

$0-10800 \mathrm{~s}$

(a)

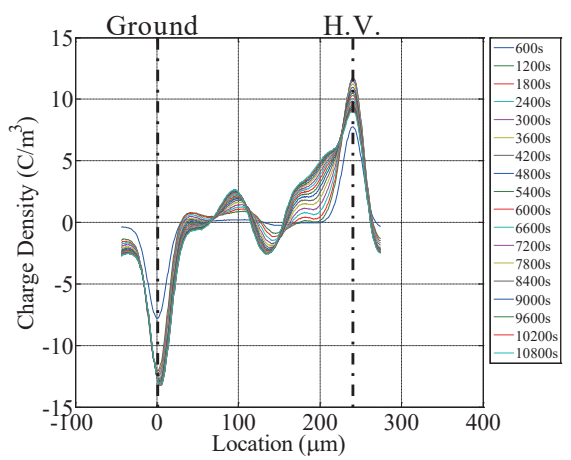

$0-10800 \mathrm{~s}$

(b)

Fig. 6. (Color online) Space charge distributions in EPR at RT under DC stress of $20 \mathrm{kV} / \mathrm{mm}$ without and with thermal gradient (1000 and $10800 \mathrm{~s})$. (a) $\Delta T=0$ and (b) $\Delta T=1.2 \mathrm{~K}$.

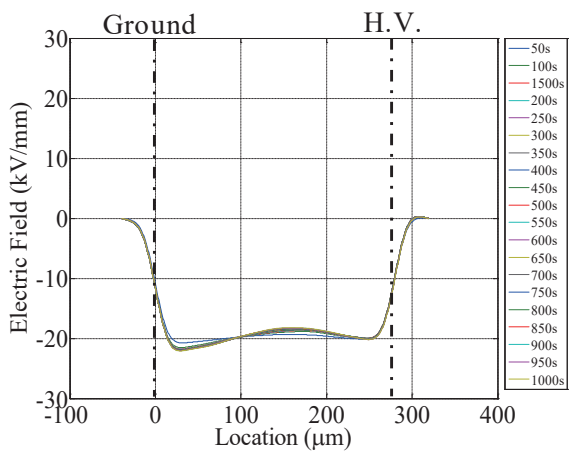

$0-1000 \mathrm{~s}$

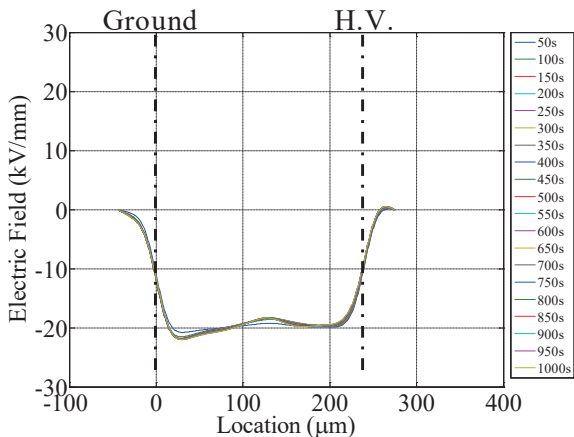

$0-1000 \mathrm{~s}$

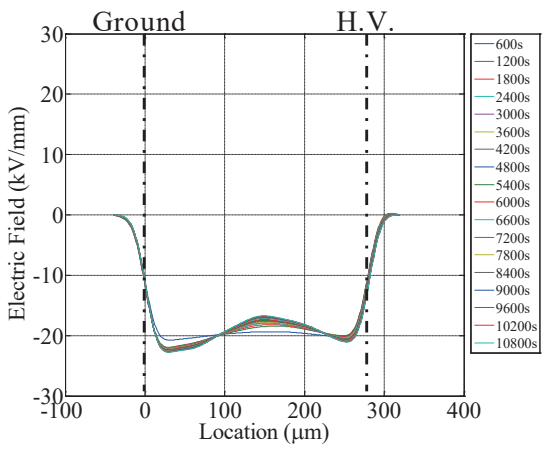

$0-10800 \mathrm{~s}$

(a)

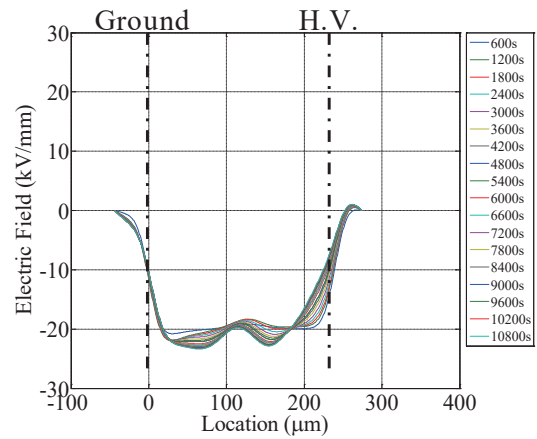

$0-10800 \mathrm{~s}$

(b)

Fig. 7. (Color online) Electric fields in EPR at RT under DC stress of $20 \mathrm{kV} / \mathrm{mm}$ without and with thermal gradient (1000 and $10800 \mathrm{~s}$ ). (a) $\Delta T=0$ and (b) $\Delta T=1.2 \mathrm{~K}$. 


\section{Discussion}

\subsection{Heterocharge accumulation in the bulk and positive homocharge injection from anode}

Heterocharge accumulation in the bulk was observed in both XLPE and EPR samples, as shown in Figs. 4-7. Moreover, positive homocharge injection from the anode was also observed in EPR, as shown in Figs. 6 and 7. Therefore, it is essential to consider the heterocharge accumulation in the bulk and positive homocharge injection from the anode. Heterocharges are formed when charge generation in the bulk dominates. When electron-hole pairs are generated in the bulk, positive charges move to the cathode side and negative charges move to the anode side, resulting in heterocharge formation.

If the thermal gradient is as large as $\Delta T=20,40$, and $60 \mathrm{~K},{ }^{(13)}$ the electric field on the side of the electrode warmed by a heater may decrease. However, there were no notable increase in electric conductivity and decrease in electric field on the warmed electrode side at $\Delta T=1.2 \mathrm{~K}$ in this experiment. For the same reason as above, there is almost no change in acoustic velocity. Therefore, the influence on the results is very small.

In general, various stabilizers such as antioxidants or metal oxides and fillers such as carbon black or silica gel are mixed in the manufacturing of XLPE and EPR. These may have promoted and accelerated the heterocharge accumulation in the bulk. It has been reported that heterocharges are formed if cable insulation contains sulfur-based antioxidants. ${ }^{(17)}$ Moreover, it is considered that excited electrons from impurities such as the residue of cross-linking agents are involved in the generation of electron-hole pairs in the bulk. ${ }^{(17)}$

It was reported that the electrical conduction of an amorphous polymer such as EPR is higher than that of a semicrystalline polymer such as XLPE because of the formation of localized energy levels. ${ }^{(18)}$ Therefore, it is important to consider energy levels to understand the space charge behavior.

\subsection{Consideration of energy levels using quantum chemical calculation}

To capture the energy levels of XLPE or EPR as a simplified model, we considered the oligomers of PE $(n=12)$ and EPR $(n=6, m=6)$, as shown in Fig. 8. In this study, we used Gaussian09 for quantum chemical calculation. The energy levels of the lowest unoccupied molecular orbital (LUMO), highest occupied molecular orbital (HOMO), bandgap $\left(E_{g}\right)$, and Fermi energy $\left(E_{F}\right)$ are shown in Table 1. Here, we used b3lyp as a functional and 6-31g as a basis set.

Actually, since XLPE and EPR are macromolecules, their $E_{g}$ values depend on the degree of polymerization. It was reported that the $E_{g}$ of PE obtained by spectroscopic measurement was $7.4 \mathrm{eV}^{(19,20)}$ Therefore, it is considered that $E_{g}$ decreases with the increase in the degree of polymerization. However, it is essential to consider also the energy levels of low molecular segments such as oligomers.

Figure 9 shows a schematic diagram of the energy levels in both electrodes and oligomers before contact. $^{(21)}$ In fact, although we used the electrode of the semiconductive layer (the work function of the semiconductive layer is inferred to be 4 to $5 \mathrm{eV}$ from graphene ${ }^{(22)}$ and the Fermi level of the semiconductive layer is obtained by subtracting the work function from the vacuum level) in the anode, the electrode was standardized to aluminum (Al). Since the work function of metal $\left(\phi_{W M}\right)$ is about $4.2 \mathrm{eV}$ in $\mathrm{Al}$, the difference in Fermi energy $\left(\Delta E_{F}\right)$ between the sample $\left(E_{F S}\right)$ and the metal 


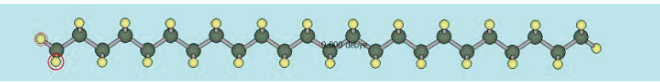

(a)

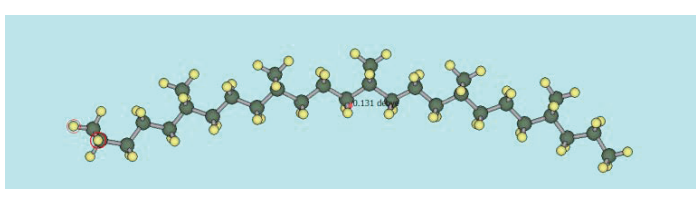

(b)

Fig. 8. (Color online) Molecular structures in PE ( $n$ $=6)$ and $\operatorname{EPR}(n=6, m=6)$. (a) $\mathrm{PE}(n=12$, electric dipole moment: 0 debye) and (b) EPR ( $n=6, m=6$, electirical dipole moment: 0.131 debye).

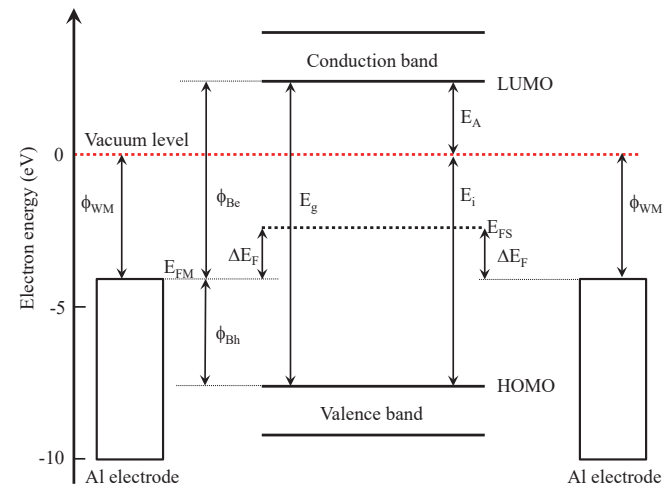

Fig. 9. (Color online) Schematic diagram of energy levels in both electrodes and oligomers before contact.

Table 1

Energy levels obtained by quantum chemical calculation.

\begin{tabular}{lcccc}
\hline \multirow{2}{*}{ Material } & \multicolumn{4}{c}{ Energy levels (eV) } \\
\cline { 2 - 5 } & LUMO & HOMO & $E_{g}$ & $E_{F}$ \\
\hline PE $(n=12)$ & 2.3889 & -7.6037 & 9.9926 & -2.6074 \\
EPR $(n=6, m=6)$ & 2.2904 & -7.4559 & 9.7463 & -2.5828 \\
\hline
\end{tabular}

$\left(E_{F M}\right)$ is about $1.6 \mathrm{eV}$. Therefore, the injection barrier of electrons $\left(\phi_{B e}\right)$ is higher than that of holes $\left(\phi_{B h}\right)$. Here, $E_{A}$ and $E_{i}$ stand for the electron affinity and ionization energy, respectively.

Figure 10 shows a schematic diagram of the energy levels in both electrodes and oligomers after contact. The electrons of the HOMO level can move to the electrode. Therefore, the surface of the oligomer is positively charged and hole levels are formed in the forbidden band. Therefore, positive charges are already injected before electric field application with only the contact.

Figure 11 shows a schematic diagram of the energy levels in both electrodes and oligomers under electric field application. The inclination in each band and Fermi energy becomes steep with increasing electric field just as in wide-gap semiconductors, and charge transport is accelerated by electric field application. Since the HOMO level is close to the Fermi energy of the anode and $\phi_{B h}$ at the anode becomes lower than that after contact, positive homocharge (hole) injection is increasingly promoted.

Figure 12 shows the space charge distributions in XLPE and EPR at RT under a DC stress of $100 \mathrm{kV} / \mathrm{mm}$ without and with a thermal gradient. These results are considered to correspond to a situation that the inclination is steep in Fig. 11. In XLPE without a thermal gradient, positive homocharges from the anode were injected immediately after DC stress application. In particular, homocharge injection was frequent after DC stress application to $500 \mathrm{~s}$. This is considered to be due to the oversupply of charges from the anode. Moreover, packetlike charges were observed and moved from the anode to the cathode in the bulk. Moreover, with a thermal gradient, homocharge injection was also frequent after DC stress application to $200 \mathrm{~s}$, and the packetlike charge transport was more active and faster than that without a thermal gradient. 


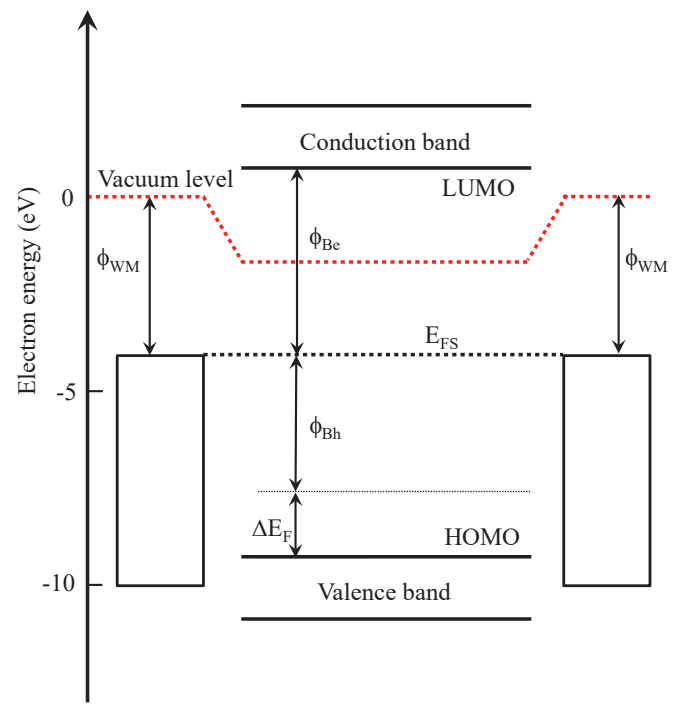

Fig. 10. (Color online) Schematic diagram of energy levels in both electrodes and oligomer after contact.

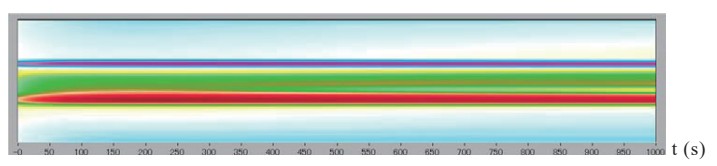

(a)

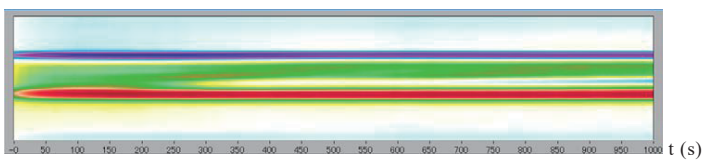

(c)

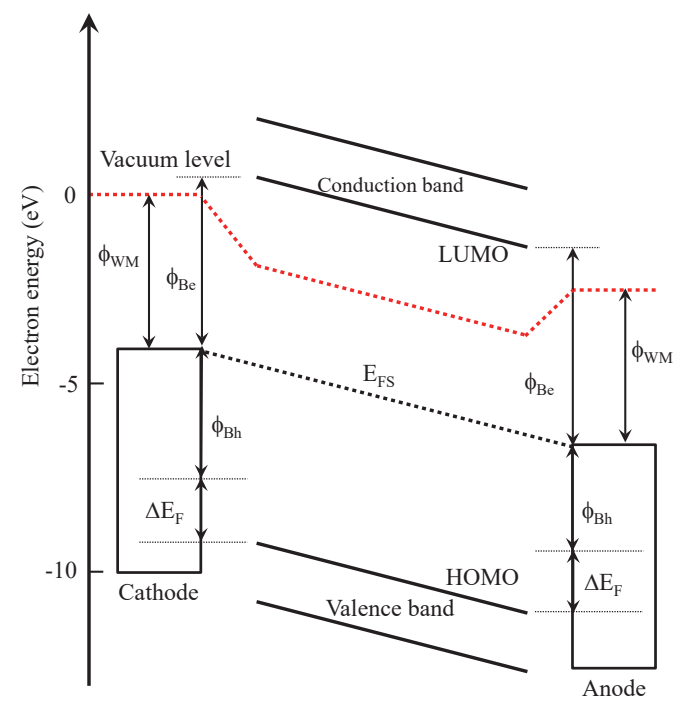

Fig. 11. (Color online) Schematic diagram of energy levels in both electrodes and oligomer under positive electric field application.

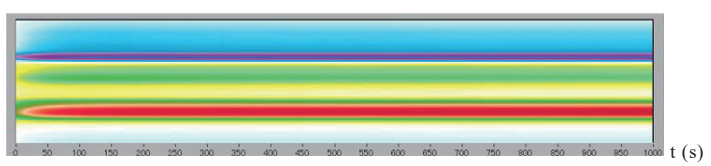

(b)

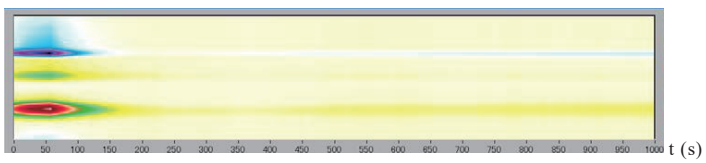

(d)

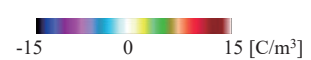

Fig. 12. (Color online) Space charge distributions in XLPE and EPR at RT under DC stress of $100 \mathrm{kV} / \mathrm{mm}$ without and with thermal gradient (1000 s). (a) XLPE, $\Delta T=0$, (b) XLPE, $\Delta T=1.2 \mathrm{~K}$, (c) EPR, $\Delta T=0$, and (d) EPR, $\Delta T=1.2 \mathrm{~K}$.

In EPR without a thermal gradient, a large number of positive homocharges were injected from the anode and a wide band of positive charges (heterocharges) appeared immediately from after DC stress application in the vicinity of the cathode. Packetlike charges were not formed in EPR. On the other hand, for one EPR sample with a thermal gradient, a breakdown took place at around $50 \mathrm{~s}$, which is considered to be caused by the oversupply of charges from the anode.

Space charge accumulations are the results of electron or hole trapping in the trap center. Therefore, it is important to evaluate physical or chemical defects and impurities. Moreover, it is considered that the energy levels of the trap center are reduced by the thermal gradient. 


\section{Conclusions}

A parallel-plate PEA system was enhanced to facilitate space charge profiling on flat samples under a thermal gradient. Such capability makes it possible to characterize new extruded HVDC cable materials in the early development phase under most relevant testing conditions to HVDC cabling where thermal gradient-driven spatial variations of electromagnetic constitutive parameters of the cable dielectric could be reproduced at a lab scale for the generation and study of space charge. Furthermore, this new system provides the direct observation of space charge injection, transport, accumulation, and recombination in solid dielectrics, as well as their spatial evolution over time for the understanding of the design stress and the aging mechanism for HVDC. The space charge behavior in model XLPE- and EPR-based HVDC samples with and without a thermal gradient has been investigated using a modified PEA system for fields at 20 and $100 \mathrm{kV} / \mathrm{mm}$. Some of the findings are as follows.

Heterocharges accumulated under a DC stress of $20 \mathrm{kV} / \mathrm{mm}$ without and with a thermal gradient in both XLPE and EPR. As a result, the field distortion was observed for both XLPE and EPR. Such charge accumulation and field distortion were enhanced by the thermal gradient.

Note that for both the model XLPE- and EPR-based HVDC samples under study, such charge accumulation and field distortion are similar in magnitude and both are actually low; in relative comparison, small quantities of heterocharges were observed at a DC stress of $20 \mathrm{kV} / \mathrm{mm}$ even within $1000 \mathrm{~s}$ in EPR, although they were not seen in XLPE.

Under a DC field of $100 \mathrm{kV} / \mathrm{mm}$, the energetic mode of packetlike charge transport from the anode to the cathode was observed in XLPE. Such an energetic mode of packetlike charge transport was further enhanced in the presence of a thermal gradient; in comparison, charge injection in EPR under the same field did not render in such packetlike charge transport, although a breakdown took place in the presence of a thermal gradient. Such breakdown took place occasionally for both XLPE and EPR, but mostly with the application of the thermal gradient. Initial quantum chemical calculation was conducted to understand the formation and nature of trapping in relation to space charge formation and transport.

\section{Acknowledgments}

The authors would like to thank Dr. S. Boggs (Nonlinear Systems, Inc.) and Professor T. Takada (Tokyo City University) for valuable discussions and comments. The authors would also like to thank Ms. J. Ronzello of The Electrical Insulation Research Center of the University of Connecticut for help with sample preparation and equipment design.

\section{References}

1 D. Fabiani, G. C. Montanari, C. Laurent, G. Teyssedre, P. H. F. Morshuis, R. Bodega, and L. A. Dissado: IEEE Electr. Insul. Mag. 23 (2007) 11.

2 S. Delpino, D. Fabiani, G. C. Montanari, C. Laurent, G. Teyssedre, P. H. F. Morshuis, R. Bodega, and L. A. Dissado: IEEE Electr. Insul. Mag. 24 (2008) 14.

3 H. E. Orton and R. Samm: IEEE Trans. Power Delivery 12 (1997) 533.

4 S. Maruyama, N. Ishii, M. Shimada, S. Kojima, H. Tanaka, M. Asano, T. Yamanaka, and S. Kawakami: Furukawa Rev. 25 (2004) 47.

5 Y. Murata, M. Sakamaki, K. Abe, Y. Inoue, S. Mashio, S. Kashiyama, O. Matsunaga, T. Igi, M. Watanabe, S. Asai, and S. Katakai: SEI Tech. Rev. 76 (2013) 55. 
6 N. Hozumi, H. Suzuki, T. Okamoto, K. Watanabe, and A. Watanabe: IEEE Trans. Dielectr. Electr. Insul. 1 (1994) 1068.

7 F. Aida, S. Wang, M. Fujita, and G. Tanimoto: IEEJ Trans. FM 117 (1997) 922 (in Japanese).

8 Y. Li, M. Yasuda, and T. Takada: IEEE Trans. Dielectr. Electr. Insul. 1 (1994) 188.

9 D. Fabiani, G. C. Montanari, C. Laurent, G. Teyssedre, P. H. F. Morshuis, R. Bodega, and L. A. Dissado: IEEE Electr. Insul. Mag. 24 (2008) 5.

10 T. L. Hanley, R. P. Burford, R. J. Fleming, and K. W. Barber: IEEE Electr. Insul. Mag. 19 (2003) 13.

11 M. Fukuma, M. Wadamori, M. Nagao, M. Kosaki, Y. Kohno, and T. Maeno: IEEJ Trans. FM 121 (2001) 80 (in Japanese).

12 W. Choo, G. Chen, and S. G. Swingler: Proc. 9th ICPADM (IEEE, Harbin, 2009) p. 946.

13 X. Chen, X. Wang, K. Wu, Z. R. Peng, Y. H. Cheng, and D. M. Tu: IEEE Trans. Dielectr. Electr. Insul. 17 (2010) 1796.

14 K. Kadowaki, Y. Nakashiki, S. Nishimoto, and I. Kitani: Proc. 2004 ICSD (IEEE, Toulouse, 2004) p. 236.

15 H. Uehara, Z. Li, Y. Cao, Q. Chen, and G. C. Montanari: 2015 Annual Report of CEIDP (IEEE, Ann Arbor, 2015) p. 138.

16 S. Ishizaki, S. Sato, Y. Tanaka, T. Fukao, and T. Takada: IEEJ Trans. FM 124 (2004) 902 (in Japanese).

17 Y. Sekii and T. Maeno: IEEE Trans. Dielectr. Electr. Insul.16 (2009) 668.

18 G. Teyssedre and C. Laurent: IEEE Trans. Dielectr. Electr. Insul. 12 (2005) 857.

19 T. Takada: 1999 Annual Report of CEIDP (IEEE, Austin, 1999) p. 1.

20 T. Tanaka: J. Appl. Phys. 44 (1973) 2430.

21 H. Uehara, Q. Chen, G. C. Montanari, Z. Li, and Y. Cao: Proc. 1st ICD (IEEE, Montpellier, 2016) p.143.

22 Y. J. Yu, Y. Zhao, S. Ryu, L. E. Brus, K. S. Kim, and P. Kim: Nano Lett. 9 (2009) 3430.

\section{About the Authors}

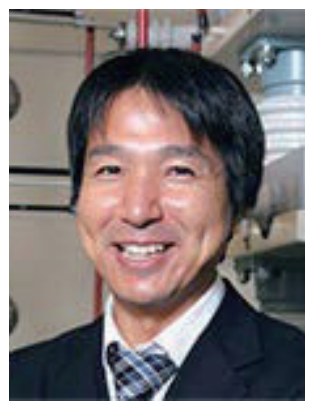

Hiroaki Uehara received his B.E., M.E., and Ph.D. degrees from Meiji University, Japan, in 1995, 1997, and 2000, respectively. In 2000, he joined the staff of the college of Engineering, Kanto Gakuin University, Japan. He was a visiting research scholar at the University of Connecticut from April 2014 to March 2015. Since 2013, he has been a professor at Kanto Gakuin University. His research interests are in insulating materials, dielectrics, and electrostatic transducers.

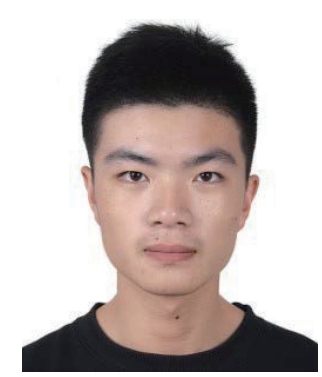

Zongze Li received his B.S. degree from Tongji University, China, in 2014. He is currently a Ph.D. student at the University of Connecticut. His research interests are in high-voltage field phenomena.

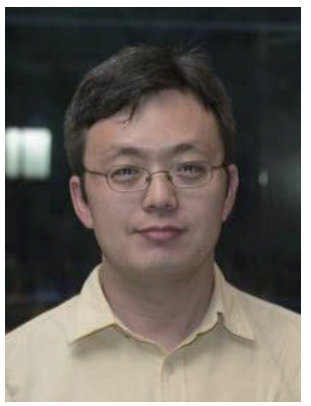

Qin Chen received his B.S. degree from Tsinghua University, China, in 2003 and his M.S. and Ph.D. degrees from Pennsylvania State University in 2006 and 2008, respectively. He is currently an electrical engineer at the GE Global Research Center. His work focused on the electro-optic, dielectric, and electromechanical properties of ferroelectric polymers. He has extensive experience in developing various ferroelectric-polymer-based devices such as tunable fiber gratings, electromechanical actuators, and energy storage capacitors. His research interests are in high-energy-density dielectric materials, tunable microwave devices, and polymeric sensors and actuators. 


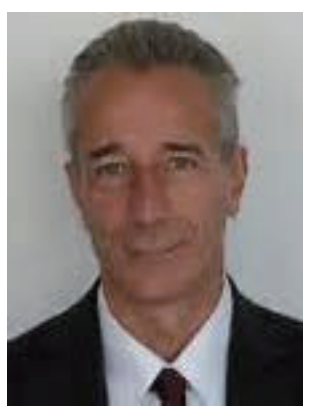

Gian Carlo Montanari is currently Full Professor of Electrical Technology at the Department of Electrical Engineering of the University of Bologna, and teaches courses on technology, reliability, and asset management. $\mathrm{He}$ has worked since 1979 in the fields of aging and endurance of solid insulating materials and systems, diagnostics of electrical systems, and innovative electrical materials (magnetics, electrets, and superconductors). He has also been engaged in the fields of power quality and energy market, power electronics, reliability and statistics of electrical systems, as well as smart grids. He is the founder and President of the spin-off Techimp, established in 1999.

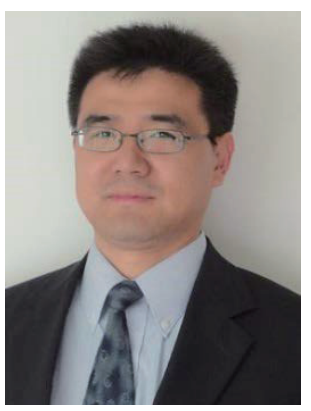

Yang Cao received his B.S. and M.S. degrees from Tongji University, China in 1992 and 1995, respectively. He received his Ph.D. degree from the University of Connecticut in 2002. He joined the GE Global Research Center as an electrical engineer for 11 years. He is presently an associate professor of the Department of Electrical and Computer Engineering and a director of the Electrical Insulation Research Center, Institute of Materials Science at the University of Connecticut. His research interests include (1) high electric field phenomena and devices, (2) polymeric nanostructured materials with game-changing characteristics for energy-efficient power conversion and renewable integrations, (3) high-voltage engineering in power and medical fields, and (4) grid asset management: diagnosis and prognosis of electrical degradation. 